\title{
Development and evaluation of a rapid and sensitive RPA assay for specific detection of Vibrio parahaemolyticus in seafood
}

\author{
Yunyun Geng ${ }^{1,2+}$, Ke Tan ${ }^{2+}$, Libing Liü ${ }^{3,4}$, Xiao Xia Sun ${ }^{3,4}$, Baohua Zhao ${ }^{2^{*}}$ and Jianchang Wang ${ }^{3,4^{*}}$ (D)
}

\begin{abstract}
Background: Vibrio parahaemolyticus (V. parahaemolyticus) is a leading cause of food poisoning and is of great importance to public health due to the frequency and seriousness of the diseases. The simple, timely and efficient detection of this pathogen is a major concern worldwide. In this study, we established a simple and rapid method based on recombinase polymerase amplification (RPA) for the determination of $V$. parahaemolyticus. According to the gyrB gene sequences of $V$. parahaemolyticus available in GenBank, specific primers and an exo probe were designed for establishing real-time recombinase polymerase amplification (real-time RPA).

Results: The real-time RPA reaction was performed successfully at $38^{\circ} \mathrm{C}$, and results were obtained within 20 min. The method only detected $V$. parahaemolyticus and did not show cross-reaction with other bacteria, exhibiting a high level of specificity. The study showed that the detection limit (LOD) of real-time RPA was $1.02 \times 10^{2}$ copies/ reaction. For artificially contaminated samples with different bacteria concentrations, $V$. parahaemolyticus could be detected within 5-12 min by real-time RPA in oyster sauce, codfish and sleeve-fish at concentrations as low as 4 CFU/25 g, 1 CFU/25 g and 7 CFU/25 g, respectively, after enrichment for $6 \mathrm{~h}$, but were detected in a minimum of 35 min by real-time PCR (Ct values between 27 and 32).
\end{abstract}

Conclusion: This study describes a simple, rapid, and reliable method for the detection of $\mathrm{V}$. parahaemolyticus, which could potentially be applied in the research laboratory and disease diagnosis.

Keywords: Vibrio parahaemolyticus, gyrB, Real-time RPA, Molecular detection

\section{Background}

Vibrio parahaemolyticus (V. parahaemolyticus) is a gram-negative, halophilic, rod-shaped bacterium belonging to the family Vibrionaceae. It is naturally present in brackish coastal environments and is frequently isolated from a variety of seafood $[1,2]$. V. parahaemolyticus is a major seafood-borne pathogen that causes gastrointestinal disorders due to ingestion of raw or undercooked seafood contaminated with this pathogen $[3,4]$. In

\footnotetext{
* Correspondence: zhaobaohua@mail.hebtu.edu.cn;

jianchangwang1225@126.com

TYunyun Geng and Ke Tan contributed equally to this work.

${ }^{2}$ College of Life Sciences, Hebei Normal University, No.20, Road E. 2nd Ring

South, Yuhua District, Shijiazhuang, Hebei Province 050024, People's Republic of China

${ }^{3}$ Center of Inspection and Quarantine, Hebei Entry-Exit Inspection and Quarantine Bureau, No.318 Hepingxilu Road, Shijiazhuang 050024, Hebei, China

Full list of author information is available at the end of the article
}

recent years, outbreaks of $V$. parahaemolyticus infections have been a significant public health concern in many countries. Additionally, it should be noted that the number of $V$. parahaemolyticus infections has increased, and their reach has widened globally during recent years [57]. V. parahaemolyticus-infected persons are characterized by an acute gastroenteritis disorder with clinical signs of diarrhea, headache, and vomiting $[4,8,9]$. Low immunity populations who become infected with $V$. parahaemolyticus may develop septicemia in severe cases [10]. Controlling the amount of $V$. parahaemolyticus in seafood is an effective way to prevent infection by this pathogen; therefore, it is important to determine the levels of $V$. parahaemolyticus in seafood [11].

Early and rapid diagnosis is crucial for the management of $V$. parahaemolyticus infection. Different diagnostic methods for $V$. parahaemolyticus infection have been reported. Conventional culturing and immunological 
methods are common techniques used for $V$. parahaemolyticus detection $[2,9,12,13]$. However, they are timeconsuming and take a few days to provide a confirmed result after numerous analytical steps [12-14]. Advancements in biotechnology have led to the development of a number of gene amplification-based molecular detection technologies for $V$. parahaemolyticus, with varying degrees of sensitivity and specificity. These include polymerase chain reaction (PCR) [1], real-time PCR [11, 15], loopmediated isothermal amplification (LAMP) [16, 17], and cross-priming amplification (CPA) [18, 19]. PCR has been widely employed for the rapid detection of $V$. parahaemolyticus. However, it is impractical for on-site application due to the expensive thermal cycling instruments required and time-consuming operation. Recently, a LAMP method was reported for rapid and sensitive detection of $V$. parahaemolyticus, but the reaction time and temperature were approximately $1 \mathrm{~h}$ and $65^{\circ} \mathrm{C}$ [16]. A simple, rapid, accurate and user-friendly platform is still needed for the early point-of-need (POD) detection of $V$. parahaemolyticus infection.

Recombinase polymerase amplification (RPA), a novel isothermal gene amplification technique, has been demonstrated to be a simple, rapid, specific, sensitive and cost-effective molecular assay to identify diverse pathogens [20-25]. The RPA process relies on three core

Table 1 Bacterial strains used in the specificity test

\begin{tabular}{|c|c|c|c|}
\hline Stains & Origin & Real-time RPA & Real-time PCR \\
\hline Vibrio parahaemolyticus & CICC 21617 & + & + \\
\hline Vibrio parahaemolyticus & ATCC 17802 & + & + \\
\hline Vibrio parahaemolyticus & Isolated in our lab & + & + \\
\hline Vibrio parahaemolyticus & Isolated in our lab & + & + \\
\hline Vibrio parahaemolyticus & Isolated in our lab & + & + \\
\hline Vibrio mimicus & CICC10474 & - & - \\
\hline Vibrio cholera & ATCC51394 & - & - \\
\hline Vibrio anguillarum & CICC10475 & - & - \\
\hline Vibrio alginolyticus & CICC21664 & - & - \\
\hline Vibrio vulnificus & CICC21615 & - & - \\
\hline Bacillus cereus & ATCC 11778 & - & - \\
\hline Bacillus cereus & CICC 10648 & - & - \\
\hline Campylobacter jejuni & ATCC 33291 & - & - \\
\hline Citrobacter freundii & ATCC 10787 & - & - \\
\hline Cronobacter sakazakii & ATCC 29544 & - & - \\
\hline Cronobacter sakazakii & ATCC 21548 & - & - \\
\hline Escherichia coli 0157:H7 & CICC 21530 & - & - \\
\hline Escherichia coli & CMCC 44102 & - & - \\
\hline Enterococcus faecalis & ATCC 29212 & - & - \\
\hline Klebsiella pneumoniae & ATCC 4352 & - & - \\
\hline Listeria monocytogenes & ATCC 19114 & - & - \\
\hline Proteus mirabilis & ATCC 29906 & - & - \\
\hline Providencia & ATCC 29944 & - & - \\
\hline Pseudomonas aeruginosa & Isolated by lab & - & - \\
\hline Salmonella typhimurium & CICC 22956 & - & - \\
\hline Serratia marcescens & ATCC 14756 & - & - \\
\hline Shigella sonnei & CICC 21679 & - & - \\
\hline Shigella sonnei & ATCC 25931 & - & - \\
\hline Shigella flexneri & CICC 21678 & - & - \\
\hline Staphylococcus aureus & ATCC 6538 & - & - \\
\hline Staphylococcus aureus & ATCC 25923 & - & - \\
\hline Yersinia enterocolitica & CICC 21609 & - & - \\
\hline
\end{tabular}

+ , positive result; - , negative result 
enzymes: a recombinase, a single-stranded DNA-binding protein (SSB) and a strand-displacing polymerase. The recombinase is capable of pairing the primer with the homologous sequence in the target DNA [25]. SSB binds to the strand of DNA displaced by the primer and stabilizes the D-loop that has formed to prevent the dissociation of primers. Finally, the strand-displacing polymerase adds bases to the $3^{\prime}$ end of the primer and primer extension occurs. When opposing primers are used, exponential amplification of the target sequence with RPA can be achieved in 20 min or less. This study describes the development and evaluation of a real-time RPA method that utilizes the fluorescent TwistAmp1 exo probe and portable instrumentation for the simple and rapid detection of $V$. parahaemolyticus.

\section{Methods}

\section{Bacterial strains and DNA extraction}

A total of $5 \mathrm{~V}$. parahaemolyticus strains, 5 other Vibrio species and 22 other bacterial strains were used to determine the specificity of the real-time RPA (Table 1). These strains were stored in our lab. Stock cultures were stored at $-80^{\circ} \mathrm{C}$ in $0.8 \mathrm{~mL}$ of Nutrient broth (Beijing Land Bridge Technology Co., Ltd., Beijing, China) and $0.2 \mathrm{~mL}$ of $80 \%$ glycerol. The DNA templates were extracted with the TIANamp Bacteria DNA Kit (Tiangen, Beijing, China). All DNA templates were stored at $-20^{\circ} \mathrm{C}$ until assayed.

\section{RPA primers and probe}

Nucleotide sequence data for $V$. parahaemolyticus strains from GenBank were aligned to identify conserved regions. According to the reference sequences of different $V$. parahaemolyticus genotypes (accession numbers: AM235735, DQ316918, FM202616, and EU051591), three pairs of primers targeting the conserved region of $\operatorname{gyrB}$ were designed. The real-time RPA primers and probes were selected by testing the combination that yielded the highest sensitivity (Table 2). Primers and exo probes were synthesized by Sangon (Sangon, Shanghai, China).

\section{Real-time RPA reactions}

Real-time RPA was carried out as describled previously $[26,27]$. The Genie III scanner device (OptiGene
Limited, West Sussex, UK) and TwistAmpTM exo kit (TwistDX, Cambridge, UK) was applied in the real-time RPA.

\section{Real-time PCR for V. parahaemolyticus}

Real-time PCR was performed on the ABI 7500 instrument as described previously [27]. Premix Ex TaqTM (Takara Co., Ltd., Dalian, China) was used in the realtime PCR, and the reaction was performed as follows: $95^{\circ} \mathrm{C}$ for $2 \mathrm{~min}$, followed by 35 cycles of $95^{\circ} \mathrm{C}$ for $10 \mathrm{~s}$, $60^{\circ} \mathrm{C}$ for $34 \mathrm{~s}$. The sequences of the primers and probes used for real-time PCR are listed in Table 2. The reporter and fluorescence quencher was marked with 6FAM (6-CarboxyFluorescein) BHQ1 (Black Hole Quencher 1) respectively.

\section{Specificity and analytical sensitivity analysis}

One hundred nanograms of $V$. parahaemolyticus genomic DNA were used as the template for the specificity analysis of the real-time RPA assay. The assay was evaluated by a panel of pathogens considered to be important in food security (Table 1).

To evaluate the real-time RPA sensitivity, genomic DNA of $V$. parahaemolyticus was diluted in a 10 -fold serial dilution to achieve DNA concentrations ranging from $1.0 \times 10^{6}$ to $1.0 \times 10^{0}$ copies $/ \mu \mathrm{L}$. One microliter of each DNA dilution was used as a template and amplified with the real-time RPA assay. Real-time RPA and PCR was tested using the standard DNA in 8 replicates. The threshold time was plotted against the molecules detected.

\section{Evaluation with artificially contaminated samples}

V. parahaemolyticus was cultured in $9 \mathrm{~mL}$ of alkaline peptone water (APW) at $37^{\circ} \mathrm{C}$ for $24 \mathrm{~h}$, harvested by centrifugation at $4000 \mathrm{rpm}$ for $20 \mathrm{~min}$ at $4{ }^{\circ} \mathrm{C}$, and washed 3 times with PBS. The final pellet was then added to oyster sauce, codfish, or sleeve-fish using 10fold serial dilutions to achieve different concentrations: 1-100 CFU/mL. The oyster sauce, codfish, and sleevefish, which were verified to be free of $V$. parahaemolyticus according to the National Standard GB 4789.7-2013 (People's Republic of China, 2013), were purchased from

Table 2 Primer and probe sequences for V. parahaemolyticus real-time PCR, RPA and real-time RPA assay

\begin{tabular}{lll}
\hline Method & Name & Sequence 5'-3'Amplication $^{\prime}$ \\
\hline Real-time RPA & RPA-FP & CGAAGAAAGCGAAACGGCAACGTCAGGCGA \\
& RPA-RP & CAGATAATTCTCACCCATCGCCGATTCAACC \\
& exo Probe & GGTTTGACAGCCGTTGTTCAGTAAAAGTGCC \\
& PCR-FP & [FAM-dT-THF-[BHQ-dTTCCAAATTCTCGAGCC \\
Real-time PCR & CGGTAGTAAACGCACTGTCAGAA \\
& PCR-RP & ACGGTAAGTTTGCGTGTGAT \\
& FAM- TGGTACTAACCATCCATCGTGGCGGTC -BHQ1 \\
\hline
\end{tabular}




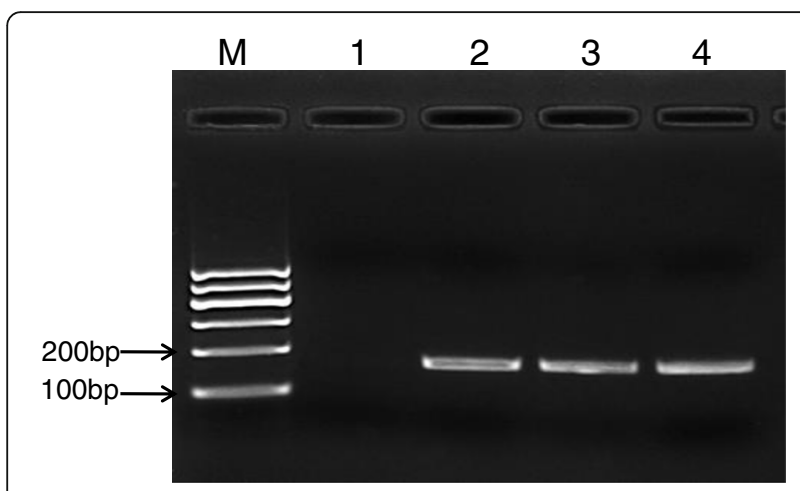

Fig. 1 Optimization of RPA reaction time. Genomic DNA of $V$. parahaemolyticus was amplified with RPA for different lengths of time, a clear DNA band with the expected size (168 bp) could be visualized by agarose gel electrophoresis after a 20 min reaction. Semi-quantification of the DNA band density using image Bio-1D software of VILBER Fusion FX5 automatic gel imaging instrument (Vilber, Marne La Vallée, France) revealed that no significant difference was observed in the product yields of $30 \mathrm{~min}$ and $40 \mathrm{~min}$ reactions. M, DNA marker, lanes $1-4$, DNA products from reactions incubated for $10 \mathrm{~min}, 20 \mathrm{~min}, 30 \mathrm{~min}$ and $40 \mathrm{~min}$

a local supermarket. Twenty five grams of oyster sauce and 4, 25 and $80 \mathrm{CFU}$ of $V$. parahaemolyticus; $25 \mathrm{~g}$ of codfish and 1, 10, and $42 \mathrm{CFU}$ of $V$. parahaemolyticus; $25 \mathrm{~g}$ of sleeve-fish samples and 7, 10, and $56 \mathrm{CFU}$ of $V$. parahaemolyticus were added into a sterile conical flask containing $225 \mathrm{~mL}$ APW (Land Bridge Technology, Beijing, China), mixed well to get homogenous samples, and then incubated for 6 or $8 \mathrm{~h}$ at $37^{\circ} \mathrm{C}$ to increase the bacterial concentrations to detectable levels. Then, $1 \mathrm{~mL}$ aliquots were collected at each time point and centrifuged $10,000 \times \mathrm{g}$ for $3 \mathrm{~min}$ at $4{ }^{\circ} \mathrm{C}$. Then, the supernatant was carefully removed and the cell pellet was washed with PBS. After centrifugation, the cell pellet was resuspended and boiled for $10 \mathrm{~min}$ to release the DNA. The resulting liquid was used as the template for the subsequent RPA assay. Each experiment was repeated at least for three times, and similar results were obtained.

\section{Statistical methods}

In order to determine the analytical sensitivity of the realtime PRA assay, a semi-log and probit regression was performed with Prism software 7.0 (Graphpad Software Inc., SanDiego, CA) and Statistical Product Service Solutions software (IBM, Armonk, NY, USA) as described previously [26].

\section{Results}

\section{Performance of RPA}

The RPA reaction was performed as previously described using $100 \mathrm{ng}$ of $V$. parahaemolyticus genomic DNA as template [22]. As shown in Fig. 1, an RPA product with the expected size (approximately $168 \mathrm{bp}$ ) was clearly visible after $20 \mathrm{~min}$ at $38^{\circ} \mathrm{C}$. Semi-quantification by measuring the DNA band density revealed that no significant difference was observed in the product yields of $20 \mathrm{~min}, 30 \mathrm{~min}$ and $40 \mathrm{~min}$ reactions (data not shown), which indicates that after $20 \mathrm{~min}$, dNTPs or

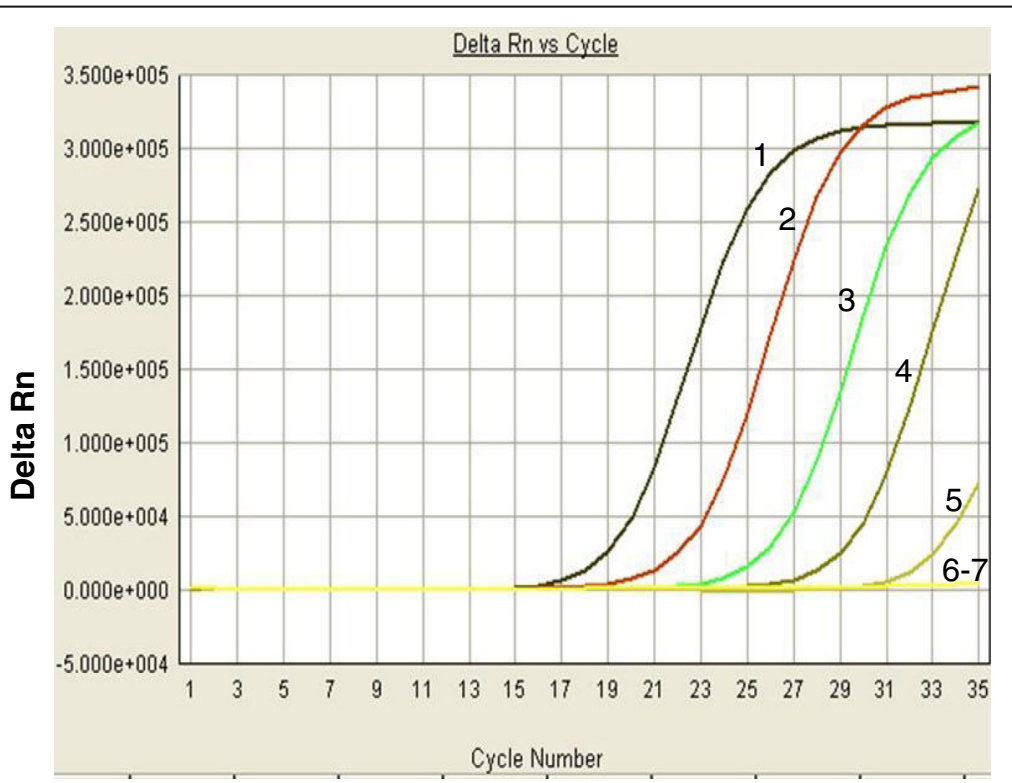

Fig. 2 Sensitivity analysis of the real-time PCR assay. Different concentrations of $V$. parahaemolyticus DNA template $\left(1.0 \times 10^{6}\right.$ to $1.0 \times 10^{0}$ copies/ reaction) were amplified by either real-time RPA or real-time PCR. As shown in this figure, the detection limit for both was $1.0 \times 10^{2}$ copies/ reaction. RPA assay is shown in panel $A$ and real-time PCR is shown in panel $B$. The concentrations used as a template for reactions 1-7 were $1.0 \times 10^{6}, 1.0 \times 10^{5}, 1.0 \times 10^{4}, 1.0 \times 10^{3}, 1.0 \times 10^{2}, 1.0 \times 10^{1}$ and $1.0 \times 10^{0}$ copies/reaction. Shown in this figure is one representative plot out of five independent reactions for real-time RPA 
other components are completely consumed. Therefore, all RPA reactions were carried out for $20 \mathrm{~min}$ for the remainder of this study.

\section{Specificity and analytical sensitivity of real-time RPA}

To assess the specificity of the real-time RPA assay under the conditions determined above, $5 \mathrm{~V}$. parahaemolyticus strains and 27 other bacterial strains that frequently contaminate food were amplified by real-time RPA. The real-time RPA reaction was performed by using an exo probe and a portable, user-friendly tube scanner. As shown in Table 1 , the $5 \mathrm{~V}$. parahaemolyticus strains were detected while the other bacterial strains, including Vibrio vulnificus, Vibrio alginolyticus, Vibrio mimicus, Vibrio anguillarum, Escherichia coli O157, S. aureus, Cronobater sakazakii, Campylobacter jejuni, Listeria monocytogenes,

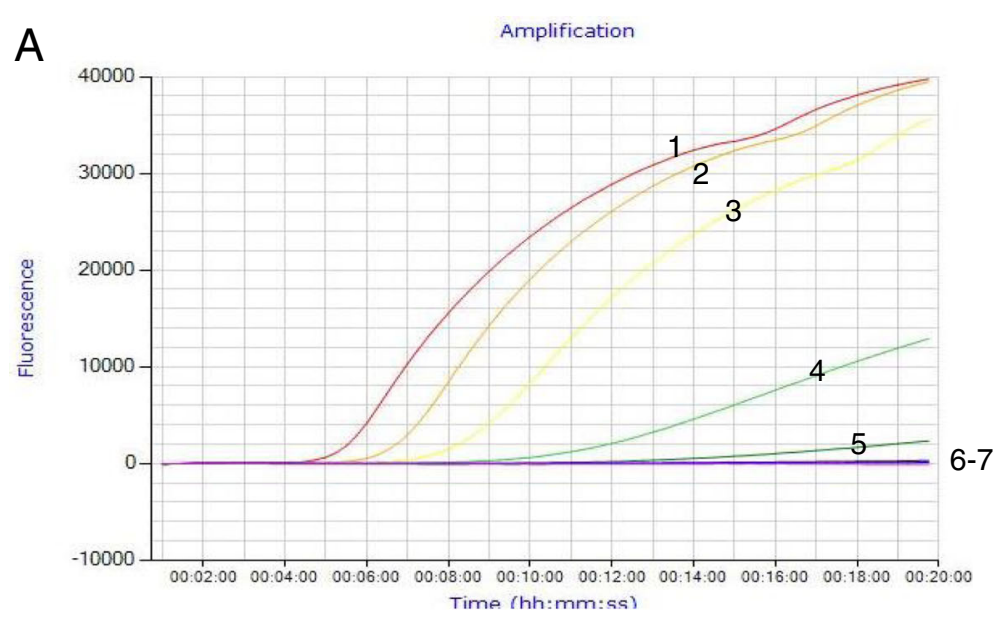

B

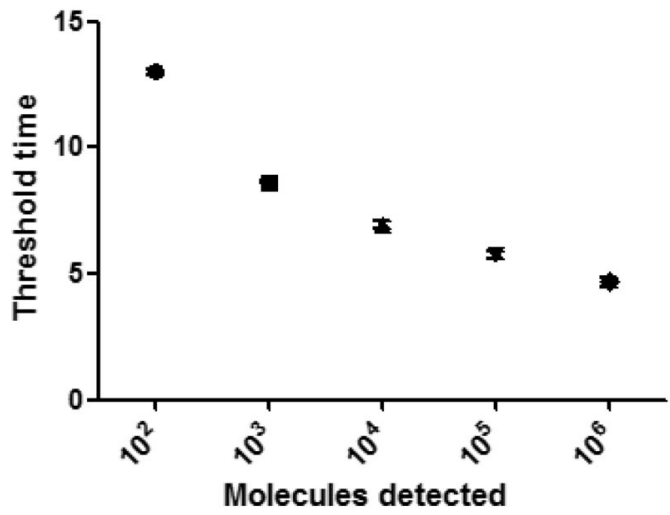

C

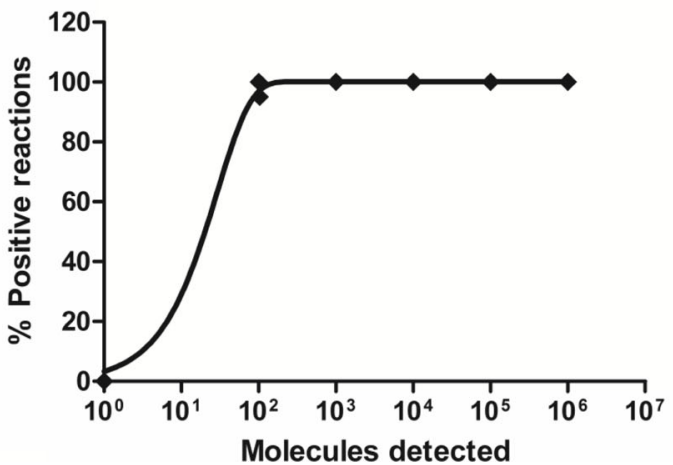

Fig. 3 Performance of real-time RPA for detection of $V$. parahaemolyticus. a. Fluorescence development over time using a dilution range of $10^{6}$ to $10^{\circ}$ copies of the standard DNA as described above. $\mathbf{b}$. Semi-logarithmic regression of the data collected from eight runs on the standard DNA using GraphPad Prism 7.0 (GraphPad Software Inc., San Diego, CA). The runtimes of real-time RPA were approximately 4.5 to $13 \mathrm{~min}$ for $10^{6}$ to $10^{2}$ copies. C. A probit regression analysis. The limit of detection of the real-time RPA was approximately $1.02 \times 10^{2}$ copies/reaction in $95 \%$ of cases and indicated by a rhomboid 
Table 3 The comparison of detection results of different methods in contaminated oyster sauce

\begin{tabular}{lllllll}
\hline $\begin{array}{l}\text { Spiked cells (CFU/25 g oyster } \\
\text { sauce) }\end{array}$ & $\begin{array}{l}\text { Enrichment time } \\
(\mathrm{h})\end{array}$ & $\begin{array}{l}\text { Real-time RPA } \\
(\mathrm{min})\end{array}$ & $\begin{array}{l}\text { Real-time PCR } \\
(\mathrm{Ct})\end{array}$ & $\begin{array}{l}\text { RPA } \\
(\mathrm{min})\end{array}$ & $\begin{array}{l}\text { GB4789.7 } \\
(\text { Day) }\end{array}$ & $\begin{array}{l}\text { Viable cell counts (CFU/ } \\
\mathrm{g})\end{array}$ \\
\hline 4 & 6 & 5.02 & 32.48 & 20 & 3 & $4.4 \times 10^{2}$ \\
& 8 & 3.35 & 21.45 & 20 & 3 & $4.8 \times 10^{2}$ \\
25 & 6 & 4.03 & 27.15 & 20 & 3 & $6.3 \times 10^{3}$ \\
& 8 & 2.88 & 20.36 & 20 & 3 & $4.1 \times 10^{3}$ \\
80 & 6 & 3.72 & 21.34 & 20 & 3 & $1.0 \times 10^{4}$ \\
& 8 & 2.72 & 16.32 & 20 & 3 & $1.0 \times 10^{4}$ \\
\hline
\end{tabular}

Shigella, Paratyphoid, and Bacillus cereus, were not. Therefore, no cross-reaction was observed for the $V$. parahaemolyticus strain examined, and the real-time RPA assay was specific for the detection of $V$. parahaemolyticus bacteria.

The analytical sensitivity of real-time RPA method was evaluated by Using $v$. parahaemolyticus genomic DNA as templates ranging from $1.0 \times 10^{6}$ to $1.0 \times 10^{0}$ copies $/ \mathrm{re}$ action. One microliter of each DNA dilution was amplified using both real-time RPA and real-time PCR. As shown in Fig. 3, the detection limit of real-time RPA was $10^{2}$ copies/reaction (panel A), while the detection limit of real-time PCR was also $10^{2}$ copies/reaction (Fig. 2). With the results of 8 complete molecular standard runs, a probit regression analysis revealed that the LOD of real-time RPA was $1.02 \times 10^{2}$ copies/reaction in $95 \%$ of cases (Fig. 3c). A semi-log regression analysis was performed for the real-time RPA. The average reaction time from 8 runs of the DNA molecular was approximately 4.5-13 min for $10^{7}-10^{4}$ copies (Fig. 3b). However, based on the real-time PCR Cq/Ct values, the real-time PCR LOD would be 1-2 log lower if the run cycles were increased to 40-45, and this would make real-time PCR more sensitive than real-time RPA assay.

\section{Evaluation with artificially contaminated samples}

The diagnostic performance of the real-time RPA assay to detect $V$. parahaemolyticus in artificially contaminated food samples was compared to that of real-time PCR. Oyster sauce, codfish, and sleeve-fish are good substrates for $V$. parahaemolyticus growth and enterotoxin production and were contaminated by $V$. parahaemolyticus with different bacteria concentrations and enrichment times as shown in Tables 3, 4 and 5. The National Standard GB 4789.7-2013 method was used as a reference assay to ensure that the food samples were successfully contaminated. The RPA and real-time RPA assays rendered a high degree of agreement (100\%) with the real-time PCR results. However, real-time RPA had an overwhelming advantage in terms of time-savings. $V$. parahaemolyticus could be detected by real-time RPA within 5-12 min in oyster sauce, codfish and sleeve-fish at concentrations as low as $4 \mathrm{CFU} / 25 \mathrm{~g}, 1 \mathrm{CFU} / 25 \mathrm{~g}$ and $7 \mathrm{CFU} / 25 \mathrm{~g}$, respectively, after enrichment for $6 \mathrm{~h}$, but detection using realtime PCR required a minimum of $35 \mathrm{~min}$ ( $\mathrm{Ct}$ values between 27 and 32). The detection time decreased in relation to increased amounts of spiked cells and enrichment time. The shortest time to obtain results by real-time RPA was $2.72 \mathrm{~min}$ (Tables 3, 4 and 5). These results strongly suggest that the real-time RPA technique has the distinct advantages of rapidity and sensitivity.

\section{Discussion}

In recent years, diseases caused by food-borne pathogens have become a significant global public health issue. $V$. parahaemolyticus is one of the major pathogens causing food-borne illness, and contamination of food products with this pathogen has become a vital concern for food safety $[9,28]$. Therefore, there is a need for rapid, specific, and reliable diagnostic techniques that can be used effectively for better detection of $V$. parahaemolyticus in seafood, environmental, and other various sample types.

In this report, the real-time RPA assay targeting the gyrB gene of $V$. parahaemolyticus was successfully

Table 4 The comparison of detection results of different methods in contaminated codfish

\begin{tabular}{|c|c|c|c|c|c|c|}
\hline Spiked cells (CFU/25 g codfish) & Enrichment time (h) & Real-time RPA (min) & Real-time PCR (Ct) & RPA (min) & GB4789.7 (Day) & $\begin{array}{l}\text { Viable cell counts } \\
\text { (CFU/g) }\end{array}$ \\
\hline \multirow[t]{2}{*}{1} & 6 & 12.02 & 30.64 & 20 & ND & 0 \\
\hline & 8 & 11.03 & 29.18 & 20 & ND & 0 \\
\hline \multirow[t]{2}{*}{10} & 6 & 6.07 & 25.12 & 20 & 3 & $5.2 \times 10^{3}$ \\
\hline & 8 & 5.37 & 20.43 & 20 & 3 & $7.1 \times 10^{3}$ \\
\hline \multirow[t]{2}{*}{42} & 6 & 5.07 & 17.45 & 20 & 3 & $2.2 \times 10^{4}$ \\
\hline & 8 & 4.20 & 17.15 & 20 & 3 & $7.2 \times 10^{4}$ \\
\hline
\end{tabular}


Table 5 The comparison of detection results of different methods in contaminated sleeve-fish

\begin{tabular}{lllllll}
\hline Spiked cells (CFU/25 g sleeve-fish) & Enrichment time (h) & Real-time RPA (min) & Real-time PCR (Ct) & RPA (min) & $\begin{array}{c}\text { GB4789.7 (Day) } \\
\text { Viable cell counts } \\
\text { (CFU/g) }\end{array}$ \\
\hline 7 & 6 & 8.15 & 27.05 & 20 & 3 & $8.9 \times 10^{2}$ \\
10 & 8 & 7.35 & 21.33 & 20 & 3 & $9.1 \times 10^{3}$ \\
& 6 & 6.33 & 25.31 & 20 & 3 & $7.5 \times 10^{2}$ \\
56 & 8 & 6.03 & 20.45 & 20 & 3 & $9.5 \times 10^{3}$ \\
& 6 & 6.05 & 17.40 & 20 & 3 & $1.0 \times 10^{5}$ \\
& 8 & 5.72 & 18.53 & 20 & 3 & $3.1 \times 10^{5}$ \\
\hline
\end{tabular}

ND Not detected

established. Conventional RPA was successfully performed at $38{ }^{\circ} \mathrm{C}$ and completed within $20 \mathrm{~min}$. The sensitivity of the real-time RPA assay was examined using serial dilutions of $V$. parahaemolyticus genomic DNA template. The limit of detection of the real-time RPA assay was $10^{2}$ copies/reaction. While the $V$. parahaemolyticus detection results by real-time RPA could be obtained in approximately $30 \mathrm{~min}$, including the time for nucleic acid extraction, the reaction time for positive samples reached up to $1 \mathrm{~h}$ when using real-time PCR [11]. In our study, the real-time RPA assay is an exact match to $99 \%$ of the sequences in GenBank. The other gyrB target sequences, approximately $1 \%$, were mismatched. Further analysis showed that the primer probes sequence used in this paper with other Vibrio parahaemolyticus had at most 3 mismatched bases, usually 1 or 2. As one of advantage, the RPA assay has demonstrated a certain tolerance to a certain length mismatch within RPA primers and exo-probe that do not influence the performance of RPA reactions, generally 3-5 mismatches according to previous research $[29,30]$. To verify the specificity of real-time RPA for the detection of $V$. parahaemolyticus, a variety of bacterial strains, listed in Table 1, were tested. The results showed that realtime RPA and real-time PCR technology both had a high degree of specificity to $V$. parahaemolyticus. However, the further sequence optimization and testing is required to ensure the assay efficiently detects all of the targeted species when it is to be applied in practice in the future.

In addition, the real-time RPA assay was also successful in the detection of artificially contaminated seafood samples, and it performed better than real-time PCR with respect to detection speed.

In recent years, a number of isothermal DNA amplification methods have been developed as a simple, rapid technique alternative to PCR-based amplification, which enable the detection of minute amounts of nucleic acid and are ideally suited to field situations [19-21]. Loopmediated isothermal amplification (LAMP) and the cross-priming amplification assay (CPA) have been adopted for rapid and sensitive detection of $V$. parahaemolyticus in seafood samples $[18,31]$. In the LAMP assay, a set of four primers is needed, and the optimum time and temperature are $60 \mathrm{~min}$ and $65^{\circ} \mathrm{C}$, respectively. Wang et al. established the real-time loop-mediated isothermal amplification technique (MERT-LAMP) for the detection of $V$. parahaemolyticus infection, which overcame the limitations posed by current LAMP technologies and allowed for real-time detection of multiple, distinct targets [16]. However, the optimal MERT-LAMP amplification temperature is $62{ }^{\circ} \mathrm{C}$ and reactions require $60 \mathrm{~min}$, which is much longer than the real-time RPA assay we used in this study. CPA was able to detect as low as $1.8 \mathrm{CFU} / \mathrm{mL}$ for pure cultures and $18 \mathrm{CFU} / \mathrm{g}$ for reconstituted samples within $60 \mathrm{~min}[18,19]$. For the real-time RPA assay described in this study, $V$. parahaemolyticus could be detected in artificially contaminated samples at concentrations as low as $1 \mathrm{CFU} / 25 \mathrm{~g}$ within $12 \mathrm{~min}$. Compared to other isothermal amplification techniques, RPA does not require initial heating for DNA denaturation, and results can be obtained in less than 12 min which didn't include the enrichment time. The real-time RPA assay has multiple advantages over other DNA amplification methods, including a quicker time-to-result for a single sample; and the potential for reduced impact of matrix-associated inhibitors [20]. RPA has been widely explored for the molecular detection of diverse pathogens, and field testing has also been achieved for Dengue virus and avian influenza A virus infection [32, 33]. Moreover, the portable POC tube scanner (Genie III, OptiGene Limited, West Sussex, United Kingdom) used in the study, weighing only 1.75 $\mathrm{kg}$ with dimensions of $25 \mathrm{~cm} \times 16.5 \mathrm{~cm} \times 8.5 \mathrm{~cm}$, is simpler than most real-time PCR machines and can be used in the field, running on battery power for an entire day.

\section{Conclusion}

In conclusion, the real-time RPA method based on an exo probe was successfully developed for the detection of $V$. parahaemolyticus. With high sensitivity and specificity, the assay could be completed within $20 \mathrm{~min}$ and the approach is easy to perform in clinical settings without a requirement for sophisticated equipment, which renders it applicable at quarantine stations, ports or sites 
of outbreaks. The effective and rapid real-time RPA assay developed in this study would be highly useful in the monitoring of $V$. parahaemolyticus infection and has the potential to be a promising alternative to real-time PCR and other isothermal methods for rapidly testing $V$. parahaemolyticus infection.

\section{Abbreviations}

ATCC: American Type Culture Collection; CFU: colony-forming units; CICC: China Center of Industrial Culture Collection; CMCC: China Center for Medical Culture Collection; CPA: cross-priming amplification; Ct: Cycle threshold; FP: Forward primer; LAMP: loop-mediated isothermal amplification; LOD: Limit of detection; PCR: Polymerase Chain Reaction; RP: Reverse primer; RPA: recombinase polymerase amplification; SSB: signal-stranded DNAbinding protein

\section{Authors' contributions}

YYG, BHZ and JCW designed and conducted the experiment. KT, LBL and XXS performed the experiments and analyzed the data. YYG drafted the manuscript. All authors read, revised, and approved the final manuscript.

\section{Funding}

This work was supported by the program of scientific research foundation in Universities of Hebei Province (QN2019025, Hebei, China); the General Administration of quality supervision, inspection and Quarantine of the People's Republic of China (2016IK107, China); and the Biology postdoctoral Science of Hebei Normal University (183717, Hebei, China);

\section{Availability of data and materials}

The dataset analyzed during the current study is available from the corresponding author on reasonable request.

\section{Ethics approval and consent to participate}

Not applicable.

\section{Consent for publication}

Not applicable

\section{Competing interests}

The authors declare that they have no conflicts of interest with the research and/ or publication of this article.

\section{Author details}

'Department of Pharmacology, Hebei University of Chinese Medicine, No.326 South Xinshi Road, Shijiazhuang 050091, Hebei, China. ${ }^{2}$ College of Life Sciences, Hebei Normal University, No.20, Road E. 2nd Ring South, Yuhua District, Shijiazhuang, Hebei Province 050024, People's Republic of China. ${ }^{3}$ Center of Inspection and Quarantine, Hebei Entry-Exit Inspection and Quarantine Bureau, No.318 Hepingxilu Road, Shijiazhuang 050024, Hebei, China. ${ }^{4}$ Hebei Academy of Inspection and Quarantine Science and Technology, No.318 Hepingxilu Road, Shijiazhuang, Hebei Province 050051, People's Republic of China.

Received: 2 January 2018 Accepted: 31 July 2019 Published online: 13 August 2019

\section{References}

1. Bauer A, Rorvik LM. A novel multiplex PCR for the identification of Vibrio parahaemolyticus, Vibrio cholerae and Vibrio vulnificus. Lett Appl Microbiol. 2007:45(4):371-5.

2. Wang R, Huang J, Zhang W, Lin G, Lian J, Jiang L, Lin H, Wang S, Wang S. Detection and identification of Vibrio parahaemolyticus by multiplex PCR and DNA-DNA hybridization on a microarray. Journal of genetics and genomics = Yi chuan xue bao. 2011;38(3):129-35.

3. Bresee JS, Widdowson MA, Monroe SS, Glass RI. Foodborne vira gastroenteritis: challenges and opportunities. Clinical infectious diseases : an official publication of the Infectious Diseases Society of America. 2002;35(6): $748-53$

4. Yingkajorn M, Mitraparp-Arthorn P, Nuanualsuwan S, Poomwised R, Kongchuay N, Khamhaeng N, Vuddhakul V. Prevalence and quantification of pathogenic Vibrio parahaemolyticus during shrimp culture in Thailand. Dis Aquat Org. 2014;112(2):103-11.

5. Centers for Disease C, Prevention. Preliminary FoodNet data on the incidence of infection with pathogens transmitted commonly through food--10 states, 2007. MMWR Morb Mortal Wkly Rep. 2008:57(14):366-70.

6. Newton A, Kendall M, Vugia DJ, Henao OL, Mahon BE. Increasing rates of vibriosis in the United States, 1996-2010: review of surveillance data from 2 systems. Clinical infectious diseases : an official publication of the Infectious Diseases Society of America. 2012;54(Suppl 5):S391-5.

7. Su YC, Liu C. Vibrio parahaemolyticus: a concern of seafood safety. Food Microbiol. 2007;24(6):549-58.

8. Bresee JS, Widdowson MA, Monroe SS, Glass RI. Foodborne viral gastroenteritis: challenges and opportunities. Clin Infect Dis. 2002;35:748-53.

9. Wang R, Zhong Y, Gu X, Yuan J, Saeed AF, Wang S. The pathogenesis, detection, and prevention of Vibrio parahaemolyticus. Front Microbiol. 2015;6:144.

10. Wang JJ, Sun WS, Jin MT, Liu HQ, Zhang W, Sun XH, Pan YJ, Zhao Y. Fate of Vibrio parahaemolyticus on shrimp after acidic electrolyzed water treatment Int J Food Microbiol. 2014;179:50-6.

11. Nordstrom JL, Vickery MC, Blackstone GM, Murray SL, DePaola A. Development of a multiplex real-time PCR assay with an internal amplification control for the detection of total and pathogenic Vibrio parahaemolyticus bacteria in oysters. Appl Environ Microbiol. 2007;73(18): 5840-7.

12. Kumar BK, Raghunath P, Devegowda D, Deekshit VK, Venugopal MN, Karunasagar I, Karunasagar I. Development of monoclonal antibody based sandwich ELISA for the rapid detection of pathogenic Vibrio parahaemolyticus in seafood. Int J Food Microbiol. 2011;145(1):244-9.

13. Liu Y, Zhang Z, Wang Y, Zhao Y, Lu Y, Xu X, Yan J, Pan Y. A highly sensitive and flexible magnetic nanoprobe labeled immunochromatographic assay platform for pathogen Vibrio parahaemolyticus. Int J Food Microbiol. 2015; 211:109-16

14. Changchai N, Saunjit S. Occurrence of Vibrio parahaemolyticus and Vibrio vulnificus in retail raw oysters from the eastern coast of Thailand. The Southeast Asian journal of tropical medicine and public health. 2014:45(3):662-9.

15. Zhang Z, Jin DZ, Zhu SR, Ye JL, Luo Y. Development of multiplex real time PCR methodology for better identification and discrimination of Vibrio cholerae and 0139 serotype. Zhonghua liu xing bing xue za zhi = Zhonghua liuxingbingxue zazhi. 2010;31(9):1026-9.

16. Wang Y, Li D, Wang Y, Li K, Ye C. Rapid and sensitive detection of Vibrio parahaemolyticus and Vibrio vulnificus by multiple endonuclease restriction real-time loop-mediated isothermal amplification technique. Molecules. 2016:21(1):E111.

17. Yamazaki W, Kumeda Y, Uemura R, Misawa N. Evaluation of a loopmediated isothermal amplification assay for rapid and simple detection of Vibrio parahaemolyticus in naturally contaminated seafood samples. Food Microbiol. 2011;28(6):1238-41.

18. Xu D, Wu X, Han J, Chen L, Ji L, Yan W, Shen Y. A cross-priming amplification assay coupled with vertical flow visualization for detection of Vibrio parahaemolyticus. Mol Cell Probes. 2015;29(6):527-30.

19. Xu G, Hu L, Zhong H, Wang H, Yusa S, Weiss TC, Romaniuk PJ, Pickerill S, You $\mathrm{Q}$. Cross priming amplification: mechanism and optimization for isothermal DNA amplification. Sci Rep. 2012;2:246.

20. Daher RK, Stewart G, Boissinot M, Bergeron MG. Recombinase polymerase amplification for diagnostic applications. Clin Chem. 2016;62(7):947-58.

21. Boyle DS, McNerney R, Teng Low H, Leader BT, Perez-Osorio AC, Meyer JC, O'Sullivan DM, Brooks DG, Piepenburg O, Forrest MS. Rapid detection of mycobacterium tuberculosis by recombinase polymerase amplification. PLoS One. 2014:9(8):e103091.

22. Liu HB, Zang YX, Du XJ, Li P, Wang S. Development of an isothermal amplification-based assay for the rapid visual detection of Salmonella bacteria. J Dairy Sci. 2017;100(9):7016-25.

23. Moore MD, Jaykus LA. Development of a recombinase polymerase amplification assay for detection of epidemic human noroviruses. Sci Rep. 2017;7:40244.

24. Sakai K, Trabasso P, Moretti ML, Mikami Y, Kamei K, Gonoi T. Identification of fungal pathogens by visible microarray system in combination with isothermal gene amplification. Mycopathologia. 2014;178(1-2):11-26.

25. Piepenburg O, Williams CH, Stemple DL, Armes NA. DNA detection using recombination proteins. PLoS Biol. 2006:4(7):e204.

26. Geng Y, Wang J, Liu L, Lu Y, Tan K, Chang YZ. Development of real-time recombinase polymerase amplification assay for rapid and sensitive detection of canine parvovirus 2. BMC Vet Res. 2017;13(1):311. 
27. Geng Y, Liu G, Liu L, Deng Q, Zhao L, Sun XX, Wang J, Zhao B, Wang J. Real-time recombinase polymerase amplification assay for the rapid and sensitive detection of campylobacter jejuni in food samples. J Microbiol Methods. 2019:157:31-6.

28. Oliver JD. The biology of Vibrio vulnificus. Microbiology spectrum. 2015;3(3).

29. Boyle DS, Lehman DA, Lillis L, Peterson D, Singhal M, Armes N, Parker M, Piepenburg O, Overbaugh J. Rapid detection of HIV-1 proviral DNA for early infant diagnosis using recombinase polymerase amplification. mBio. 2013; $4(2)$.

30. Abd El Wahed A, El-Deeb A, El-Tholoth M, Abd El Kader H, Ahmed A, Hassan S, Hoffmann B, Haas B, Shalaby MA, Hufert FT, et al. A portable reverse transcription recombinase polymerase amplification assay for rapid detection of foot-and-mouth disease virus. PLoS One. 2013;8(8):e71642.

31. Chen S, Ge B. Development of a toxR-based loop-mediated isothermal amplification assay for detecting Vibrio parahaemolyticus. BMC Microbiol. 2010;10:41.

32. Abd El Wahed A, Patel P, Faye O, Thaloengsok $S$, Heidenreich $D$

Matangkasombut P, Manopwisedjaroen K, Sakuntabhai A, Sall AA, Hufert FT, et al. Recombinase polymerase amplification assay for rapid diagnostics of dengue infection. PLoS One. 2015;10(6):e0129682.

33. Abd El Wahed A, Weidmann M, Hufert FT. Diagnostics-in-a-suitcase: development of a portable and rapid assay for the detection of the emerging avian influenza a (H7N9) virus. Journal of clinical virology : the official publication of the Pan American Society for Clinical Virology. 2015; 69:16-21.

\section{Publisher's Note}

Springer Nature remains neutral with regard to jurisdictional claims in published maps and institutional affiliations.

Ready to submit your research? Choose BMC and benefit from:

- fast, convenient online submission

- thorough peer review by experienced researchers in your field

- rapid publication on acceptance

- support for research data, including large and complex data types

- gold Open Access which fosters wider collaboration and increased citations

- maximum visibility for your research: over $100 \mathrm{M}$ website views per year

At $\mathrm{BMC}$, research is always in progress.

Learn more biomedcentral.com/submissions 